\title{
CYTOLOGICAL GRADING OF DUCTAL CARCINOMA OF BREAST IN FINE NEEDLE ASPIRATES AND ITS CORRELATION WITH HISTOLOGICAL GRADING
}

\author{
K. Hidhaya Fathima ${ }^{1}$, J. Nilavu ${ }^{2}$ \\ ${ }^{1}$ Assistant Professor, Department of Pathology, Tirunelveli Medical College. \\ ${ }^{2}$ Assistant Professor, Department of Pathology, Villupuram Medical College.
}

ABSTRACT

\section{BACKGROUND}

Grading of fine needle aspirates of breast carcinoma is useful for selection of appropriate therapy, especially for planning neoadjuvant chemotherapy.

The aim of this study is to evaluate the cytological grading by Robinson method in FNAC samples and to correlate it with the well-known prognostic factor. Histological grading system proposed by Nottingham Modification of Bloom-Richardson. The cytologic grading system was also correlated with the axillary lymph node status.

\section{MATERIALS AND METHODS}

This descriptive study was done on fifty female patients with breast lump in Department of Pathology in Coimbatore Medical College. Cytological samples were stained using $\mathrm{H}$ and E stain and Papanicolaou stain. Cytologic grading was done by Robinson method and compared with Nottingham modification of Scarff-Bloom-Richardson method histologic grading system.

\section{RESULTS}

Most of the tumours were Grade 2 tumours (46\%). The concordance between cytological grade and histological grade was $86 \%$, nearly 43 out of 50 cases. The remaining 7 cases (14\%) showed discrepancy. Among these cases, five were upgraded and two were downgraded. The association between cytological grade and histological grade were statistically significant ( $p<0.05)$. The Lymph node positivity was noted among 37.5\%,52.17\% and 72.7\% of Grade 1, 2 and 3 tumours respectively. High grade tumours show increased nodal positivity. The association between cytological grade and lymph node status was not statistically significant $(\mathrm{p}>$ 0.05). Most of the tumours were T2 $(2-5 \mathrm{~cm})$. The association between tumour size and cytologic grade was not statistically significant $(\mathrm{p}>0.05)$. Most of the tumours were located in outer quadrant $50 \%$, higher grade tumours in central quadrant.

\section{CONCLUSION}

This concludes that cytological grading should be included in all FNAC reports of ductal carcinoma of breast, so that appropriate decision can be made regarding the neoadjuvant chemotherapy can be taken and overtreatment of low-grade carcinomas can be avoided.

\section{KEYWORDS}

FNAC, Breast Carcinoma, Cytological Grading, Nottingham Modification of Scarff-Bloom-Richardson Method.

HOW TO CITE THIS ARTICLE: Fathima KH, Nilavu J. Cytological grading of ductal carcinoma of breast in fine needle aspirates and its correlation with histological grading. J. Evolution Med. Dent. Sci. 2017;6(84):5846-5852, DOI: 10.14260/jemds/2017/1270

\section{BACKGROUND}

Breast cancer is the second most common cancer in females worldwide accounting for about 1.4 million cases each year. Breast cancer is the second leading cause of cancer related deaths in females all over the world. ${ }^{1}$ As against an estimated 48,170 women who died of breast cancer in 2007, the number reached 50,000 in 2010 which raised to 50,821 by 2011. As per study conducted in Coimbatore, percentage of occurrence of breast cancer was found to be 20.3 out of 1 lakh women.

Fine needle aspiration cytology is being increasingly used to determine the benign and malignant nature of the lesion. ${ }^{2}$ Attempts have been made to determine various prognostic parameters on FNA material to determine the best therapy. ${ }^{3}$

'Financial or Other Competing Interest': None.

Submission 14-09-2017, Peer Review 08-10-2017,

Acceptance 14-10-2017, Published 19-10-2017.

Corresponding Author:

K. Hidhaya Fathima,

\#14, Balaji Nagar, High Ground, Tirunelveli.

E-mail: drhiditvmc@yahoo.co.in

DOI: $10.14260 / \mathrm{jemds} / 2017 / 1270$
It is desirable to grade the tumour, while is still in vivo for the selection of appropriate therapy. The significance of histological grading was already accepted and found to be a useful supplementary prognostic parameter. So when a significant association was established between cytologic and histologic grading systems, cytologic grading may be helpful to decide the level of treatment.

\section{MATERIALS AND METHODS}

This descriptive study was carried out in 50 cases of invasive duct carcinoma of breast for a period of one year from August 2011 - July 2012. This study was done in Department of Pathology, Coimbatore Medical College Hospital, Coimbatore. In all these cases, the cytological diagnosis were confirmed by histological examination.

\section{Inclusion Criteria}

Female patients with palpable breast mass.

\section{Exclusion Criteria}

Patients who have received neoadjuvant chemotherapy or radiotherapy. Patients whose cytological diagnosis does not have histological confirmation. Male patients with ductal carcinoma of breast. 


\section{Data Collection}

Female patients with palpable breast lesions were selected. The patients were classified into three categories based on age-

1. Pre-menopausal $(<47)$.

2. Menopausal (47 - 52).

3. Post-menopausal $(>52)$. The written consent for FNAC was obtained from the patient.

\section{FNA Procedure}

The FNA procedure was done by the Faculty and Postgraduates of Department of Pathology, Coimbatore Medical College using 23-gauge needle with or without $10 \mathrm{~mL}$ disposable syringe by non-aspiration technique. The aspirated material was expressed on slide and wet fixed and stained with Papanicolaou method and Haematoxylin and eosin.

The stained smears were evaluated and diagnosed as ductal carcinoma of breast when there is definite features of malignancy like cellular dissociation, nuclear pleomorphism, nuclear abnormalities, absence of bare nuclei. Special variants of ductal carcinoma were also included. These slides were graded based on Robinson grading system. Those smears with suspicious cell clumps and features suggestive of ductal hyperplasia were excluded.

Modified radical mastectomy specimens including axillary clearance fixed in $10 \%$ formalin were obtained. The tumour size and the tumour location were assessed in the gross specimens. Paraffin-embedded sections obtained by routine procedures were cut at thickness of 3 microns using Leica microtome. These slides were routinely stained with haematoxylin and eosin stain.

The slides were evaluated and diagnosis of invasive duct carcinoma was confirmed. Special types of ductal carcinoma other than classical invasive duct carcinoma NOS were also included in this study. Histological grading was done using Nottingham modification of Scarff-Bloom-Richardson method.

The axillary lymph nodes received were also processed in a similar manner and assessed for metastasis. Statistical analysis was done to examine the degree of association between the cytologic and histologic grading system using Chi-square test. Also, the tumour grades were correlated with other prognostic factors which includes age of the patient, tumour size and lymph node status independently. The $\mathrm{P}$ value $<0.05$ was considered to be statistically significant.

\section{OBSERVATIONS AND RESULTS}

A total of 50 cases were studied and the following observations were obtained.

\section{Age Distribution}

The patient's age ranges from 32 - 86 years with a mean age of 52.52 years. The minimum age and the maximum age reported in the present study was 32 and 86 years respectively. According to this table, majority of cases belong to the post-menopausal age group (44\%); $38 \%$ of case in premenopausal age group and the $18 \%$ of cases in the menopausal age group.

\section{Tumour Size}

The cases were divided into three categories based on tumour size according to TNM staging. Of these fifty patients four $(8 \%)$ of them have tumour size $<2 \mathrm{~cm}$, thirty $(60 \%)$ patients have tumour size between 2 and $5 \mathrm{~cm}$, sixteen (32\%) of them have tumour size $>5 \mathrm{~cm}$.

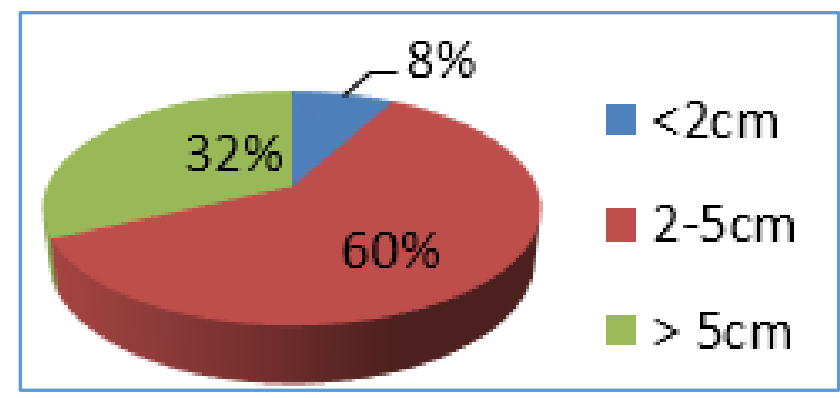

Chart 1. Distribution of Cases according to Tumour Size

\section{Lymph Node Status}

The lymph node status was classified into positive and negative nodes. Of the fifty cases, twenty four (48\%) cases were node negative. Among the twenty six node positive cases, ten cases had 1 - 3 nodes, fourteen cases had 4 - 9 nodes and two cases had more than 10 nodes.

\section{Tumour Location}

According to the location of the tumour, they have been classified into outer, inner and central quadrant. In this study, majority of the cases were located in the outer quadrant (50\%) followed by central quadrant (30\%) and inner quadrant.

\section{Cytological Grade}

According to Robinson grading system, the cytological samples were graded into three groups.

\begin{tabular}{|c|c|}
\hline Cytologic Grade & Number \\
\hline Grade 1 & $16(32 \%)$ \\
\hline Grade 2 & $23(46 \%)$ \\
\hline Grade 3 & $11(22 \%)$ \\
\hline
\end{tabular}

Table 1. Distribution of Cases with Regard to Cytological Grade

Of the fifty cases, majority of the cases belong to Grade 2 (46\%) followed by Grade 1 (32\%) and Grade 3 (22\%) with 11 cases. Few special types including mucinous carcinoma (Grade 1), papillary carcinoma (Grade 2), one with plasmacytoid morphology (Grade 1). One of the ductal carcinoma with Grade 2 nuclear features show plenty of lymphocytes in the background.

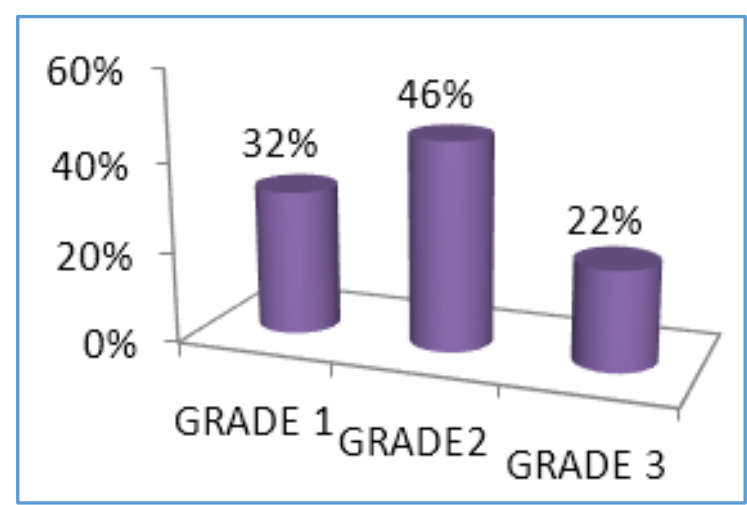

Chart 2. Distribution of Cases with Regard to Cytological Grade 


\section{Histological Grade}

According to Nottingham modification of Scarff-BloomRichardson grading, fifty cases were categorised under three categories.

\begin{tabular}{|c|c|}
\hline Histologic Grade & Number (\%) \\
\hline Grade 1 & $14(28 \%)$ \\
\hline Grade 2 & $23(46 \%)$ \\
\hline Grade 3 & $13(26 \%)$ \\
\hline \multicolumn{2}{|c|}{ Table 2. Distribution of Cases with Regard } \\
to Histological Grade
\end{tabular}

In this study of fifty cases, majority of tumours were Grade 2 tumours followed by Grade 1 and Grade 3. Among these fifty cases, there were three special types of invasive duct carcinoma and the rest of the forty-seven cases were invasive duct carcinoma, NOS. The special types include mucinous carcinoma under Grade 1, papillary carcinoma under Grade 2. One of the Grade 2 invasive ductal carcinoma NOS show neuroendocrine differentiation. One of the Grade 3 ductal carcinoma diagnosed in cytologic samples as ductal carcinoma with plenty of lymphocytes in the background was diagnosed to be atypical medullary carcinoma.

\section{Correlation of Cytologic Grade with Histologic Grade}

The cytological grade of the tumour was correlated to the histologic grade to assess the concordance between the two grading systems.

\begin{tabular}{|c|c|c|c|c|c|}
\hline & \multicolumn{3}{|c|}{ Histologic Grade } & \multirow{2}{*}{ TOTAL } \\
\hline & & 1 & 2 & 3 & \\
\hline \multirow{3}{*}{$\begin{array}{l}\text { Cytologic } \\
\text { Grade }\end{array}$} & 1 & $12(75 \%)$ & $2(12.5 \%)$ & $2(12.5 \%)$ & 16 \\
\hline & 2 & $1(4.35 \%)$ & $21(91.3 \%)$ & $1(4.35 \%)$ & 23 \\
\hline & 3 & $1(9.09 \%)$ & 0 & $\begin{array}{c}10 \\
(90.9 \%)\end{array}$ & 11 \\
\hline \multicolumn{3}{|c|}{14} & 23 & 13 & 50 \\
\hline \multicolumn{6}{|c|}{$\begin{array}{l}\text { Table 3. Correlation of Cytologic Grade } \\
\text { with Histologic Grade }\end{array}$} \\
\hline
\end{tabular}

Chi-Square Total $=60.04 \mathrm{DF}=4$ Significant at $1 \%$ level $(\mathrm{P}<$ 0.01). Kappa value $=0.782$

In this study of fifty cases, forty three cases (86\%) show concordance between cytologic and histologic grading systems. Rest of the seven cases (14\%) show discrepancy. Among seven 4 were Grade 1, 2 were Grade 2 and 1 were Grade 3.

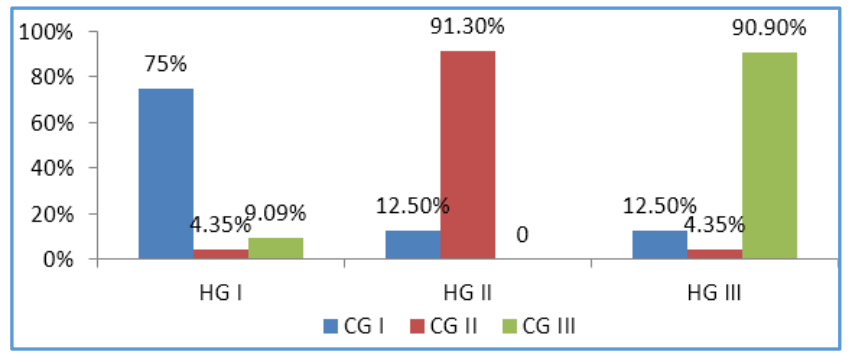

Chart 3. Correlation of Cytologic Grade with Histologic Grade

\section{Correlation of Cytologic Grade with Age}

The cytological grade was compared with the age of the patient. Grade 3 tumours was seen to occur in younger age group (63.62\%), which indicates aggressiveness of tumour in young age group. The minimum age reported was 32 years, who shows Grade 3 in both cytology samples and histology specimen. The cytologic grade did not show a significant association with age of the patient $(p>0.05)$.

\section{Correlation of Cytologic Grade with Tumour Size}

The cytologic grade was correlated with the tumour size. Majority of tumours have a size of $2-5 \mathrm{~cm}$. Tumours in all grades have a size of $2-5 \mathrm{~cm}$ with $56.25 \%$ in Grade 1, $56.52 \%$ in Grade 2 and $72.72 \%$ in Grade 3 tumours respectively.

Correlation of Cytologic Grade with Lymph Node Status The cytologic grade was correlated with the lymph node status to see whether high-grade tumours were associated with increased lymph node positivity.

\begin{tabular}{|c|c|c|c|}
\hline $\begin{array}{c}\text { Cytologic } \\
\text { Grade }\end{array}$ & \multicolumn{2}{|c|}{ Lymph Node Status } & Total \\
\hline & Negative Nodes & Positive Nodes & \\
\hline Grade 1 & $10(62.5 \%)$ & $6(37.5 \%)$ & 16 \\
\hline Grade 2 & $11(47.83 \%)$ & $12(52.17 \%)$ & 23 \\
\hline Grade 3 & $3(27.8 \%)$ & $8(72.7 \%)$ & 11 \\
\hline
\end{tabular}

All the lymph nodes mentioned here were axillary lymph nodes. No cervical and internal mammary nodes were obtained. Lymph node metastasis was observed in 6 (37.5\%) of Grade 1, 12 (52.17\%) of Grade 2 and 88 (72.7\%) of Grade 3 tumours.

Correlation of Cytological Grade with the Tumour Location

Out of the fifty cases, most of Grade 1 and Grade 2 tumours were in outer quadrant with $62.5 \%$ and $47.82 \%$ respectively, whereas most of Grade 3 tumours were in the central quadrant (54.54\%).

\section{Correlation of lymph Node Status with Tumour Size}

The lymph node status was correlated with the tumour size to see whether increase in tumour size was associated with increase in lymph node positivity.

Most of the tumours with positive lymph nodes have a size between $2-5 \mathrm{~cm}$. Maximum number of positive nodes was reported to be 18 and 15 , and corresponding tumour size were 8.5 and $7 \mathrm{~cm}$ respectively. In Mucinous carcinoma though the size was $>10 \mathrm{~cm}$, all the twelve nodes were negative indicating a favourable prognosis. There was no significant association between the tumour size and lymph node status $(\mathrm{p}>0.05)$.

\section{Grade I}

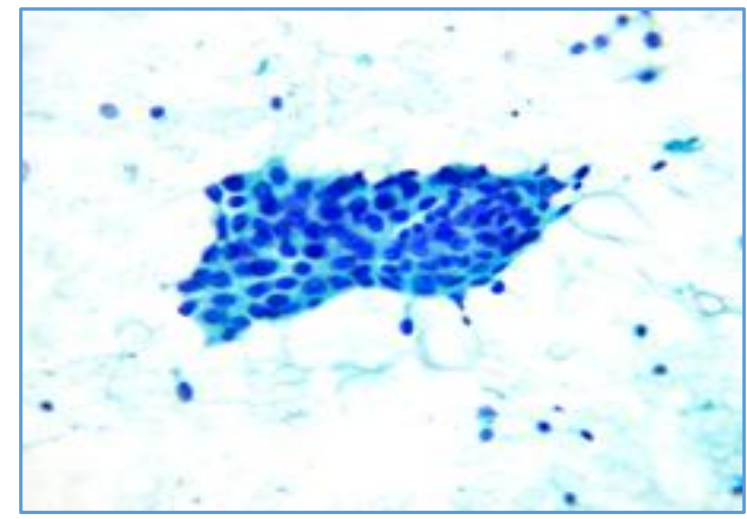

Figure 1. Monomorphic Epithelial Cells with Smooth Nuclear Margins and Inconspicuous Nucleoli (FNAC-Pap Stain) $10 \mathrm{X}$ 


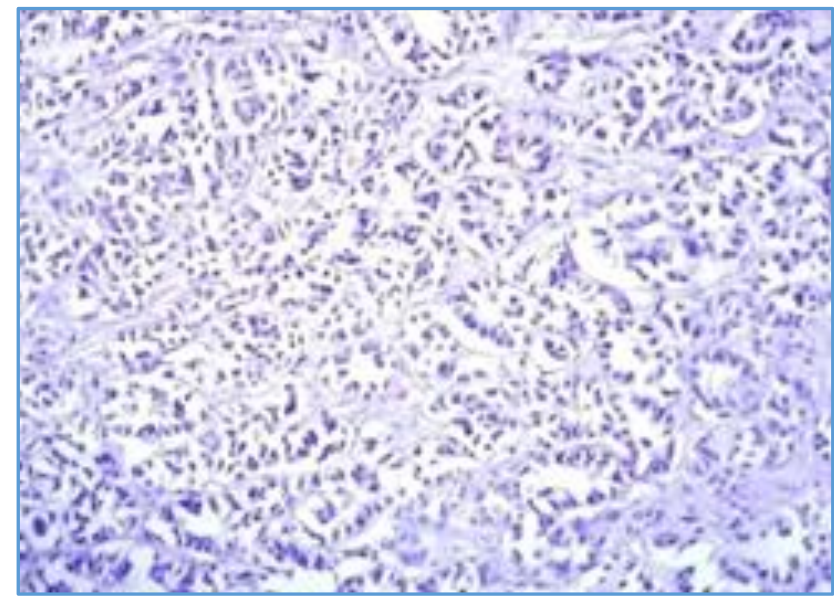

Figure 2. Tumour show $>75 \%$ Tubule Formation (HPE-H and E) 10X

Grade II

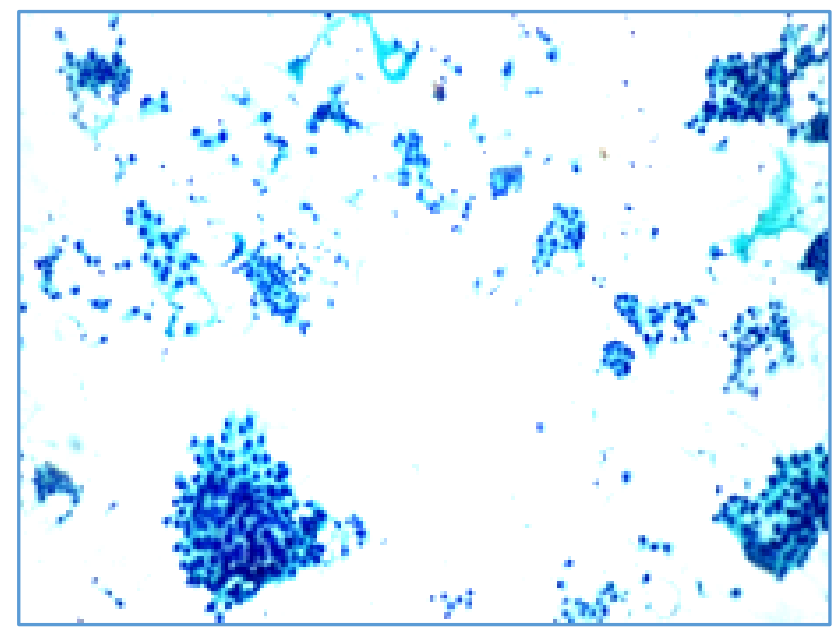

Figure 3. Malignant Epithelial Cells arranged Singly and in Clusters with Mild Pleomorphism (FNAC-Pap Stain) 10X

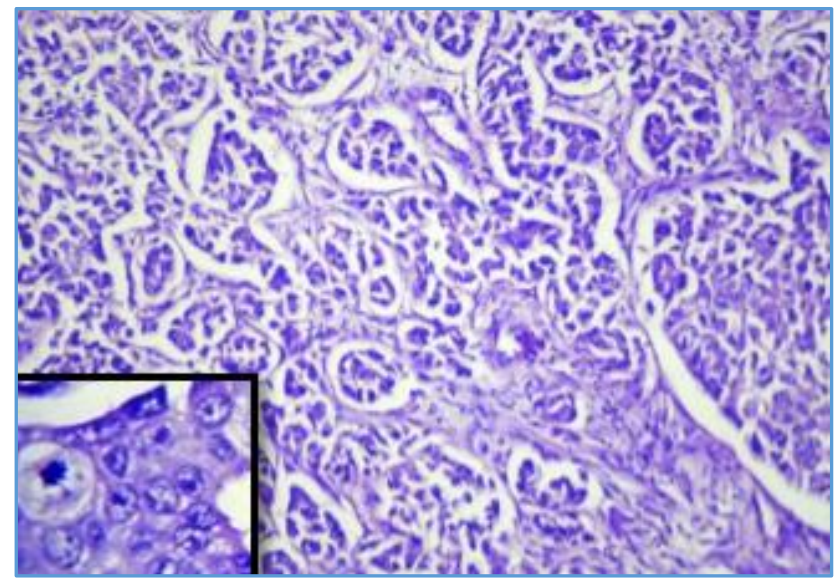

Figure 4. Tumour shows Few Tubules (Arrow) in this Field

(HPE-H and E) 10X. Inset shows Vesicular Nuclei and visible Nucleoli with One Mitotic Figure
Grade III

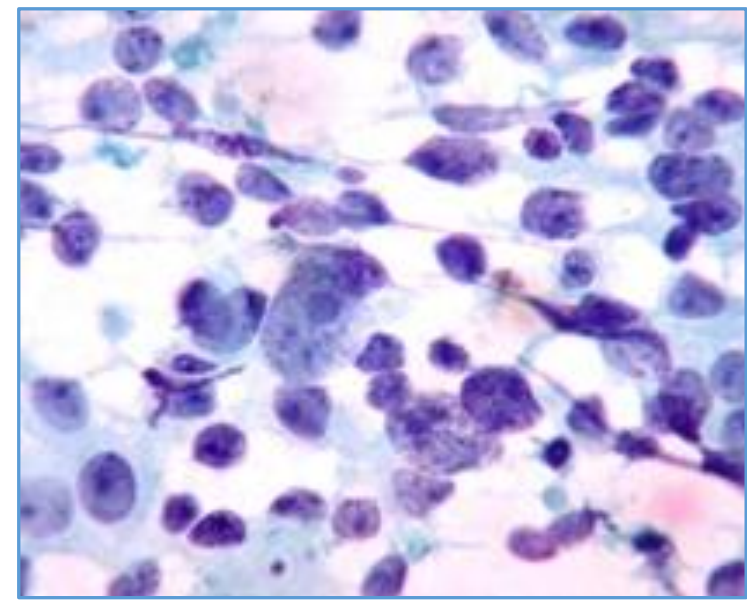

Figure 5. Malignant Epithelial Cells arranged Singly with Marked Nuclear Pleomorphism with Prominent Nucleoli (FNAC-Pap) 40X

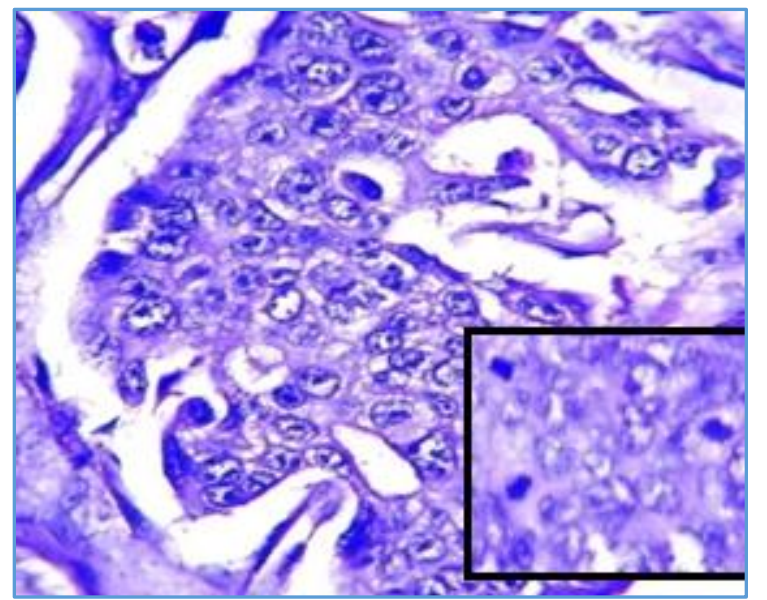

Figure 6. Large Pleomorphic Cells with Prominent Nucleoli. Inset shows Three Mitotic Figures in One Field (HPE- H and E) 40X

Special Types/ Mucinous Carcinoma

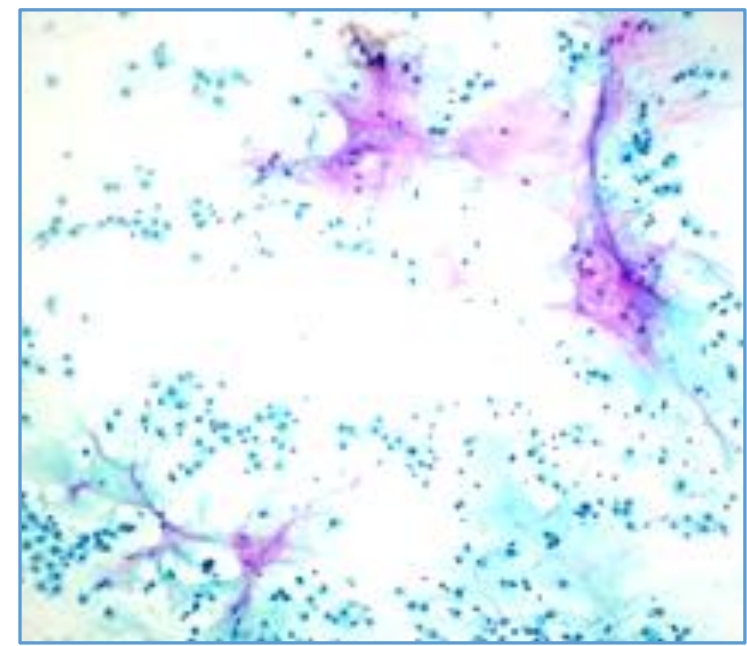

Figure 7. Tumour Cells in a Background of Mucin with Mild Atypia (FNAC- Pap) 10X 


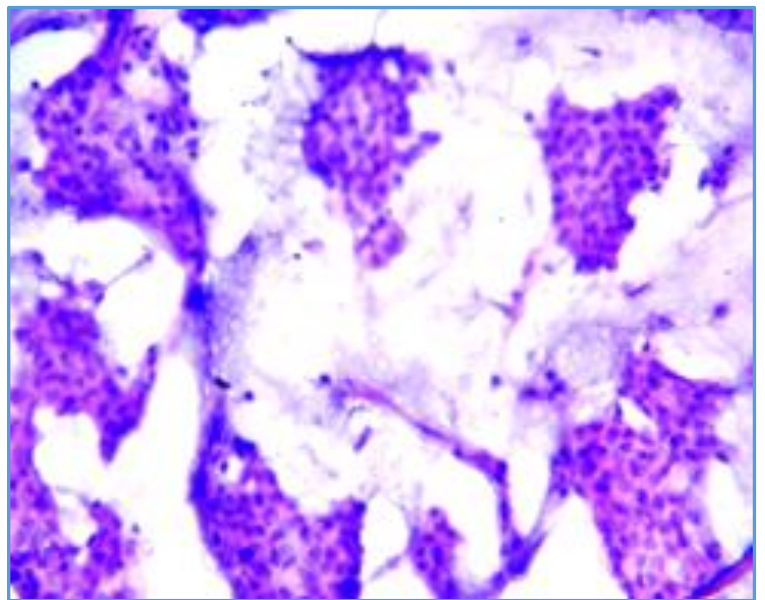

Figure 8. Nest of Tumour Cells in a Pool of Extracellular Mucin with Small Dark Nuclei showing Mild Pleomorphism (HPE- H and E) 40X (HPE: 186/12)

\section{Papillary Carcinoma}

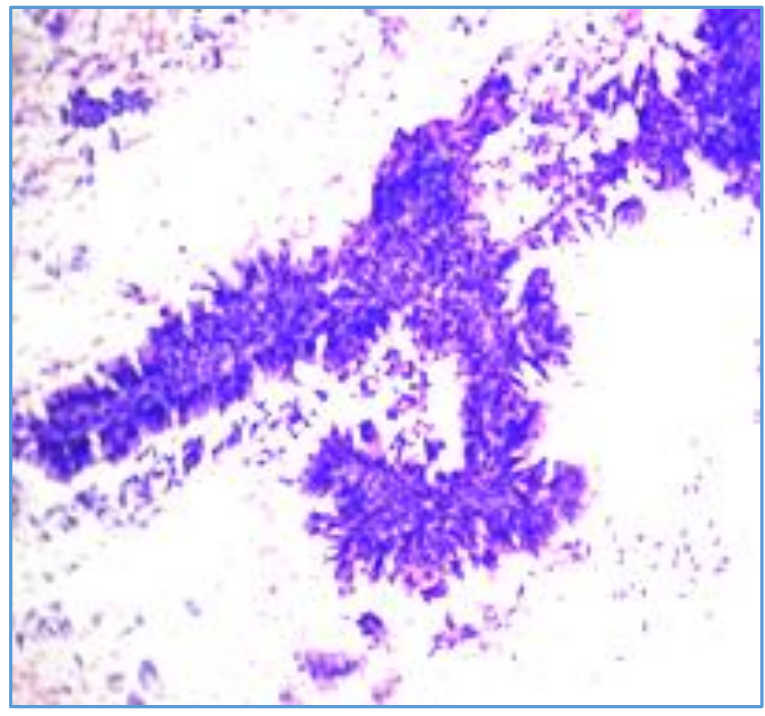

Figure 9. Branching Papillae with Fibrovascular Core Lined by Atypical Epithelial Cells (FNAC- H and E)

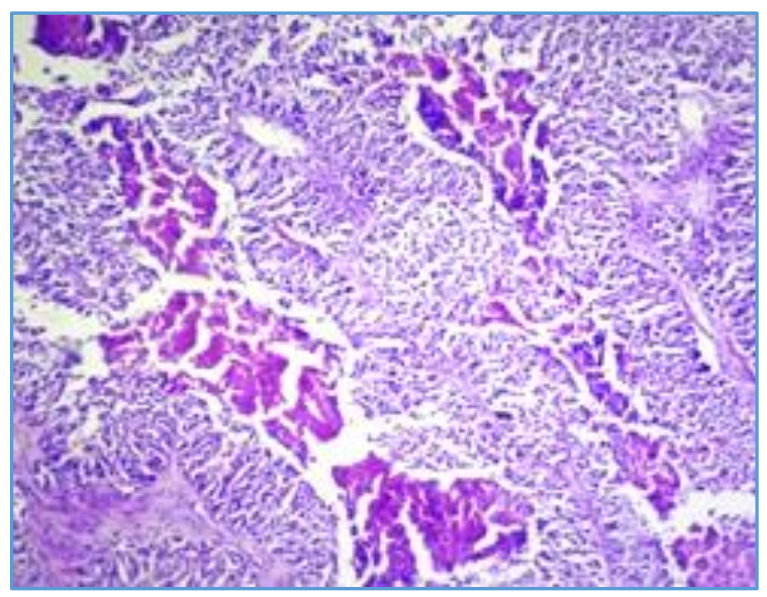

Figure 10. Papillary Structures with Fibrovascular Core (HPE- H and E) 10X

\section{DISCUSSION}

Breast carcinoma is a malignant disease with a variable prognosis. Assessment of prognostic parameters is of growing interest in recent days. The parameters consist of tumour size, lymph node status, ${ }^{4}$ oestrogen receptor status, ${ }^{5}$ histological grading and cell proliferation index. All these features have been well studied on surgical specimens; however, evaluation of prognostic factors and grading of cytologic samples have not been included in the routine practice. Cytologic grading is valuable, feasible and reproducible. This method also depicts the intrinsic characteristics of the tumour as well as its prognosis.

The need for prognostic grading in cytological samples of breast cancers is to identify rapidly growing tumours, which were more responsive to chemotherapy and better suited to pre-treatment with tamoxifen. Overtreatment of slow growing tumours was also reduced. Assessment of biological aggressiveness by cytological grading without removing the tumour would therefore be of immense value.

In this present study, predominant tumours were Grade 2 (46\%) followed by Grade 1 (32\%) and Grade 3 (22\%) in cytological samples. A study by Robles et al $^{6}$ showed a similar result, the predominant tumours were Grade 2 (39\%) followed by Grade 1 (36\%) and then Grade 3 (25\%). Studies done by Dash et al,7 Chabbra et $\mathrm{al}^{8}$ and Robinson et al also showed similar results. However, in a study by Wani et $\mathrm{al}^{9}$ Grade 3 forms the second largest group as against to Grade 1 in this study. This shows the increasing awareness and early presentation of the patients in our setup.

This study showed a high degree of concordance (86\%) between the cytological and histological grading systems. Khan et $\mathrm{al}^{10}$ showed a concordance value of $84 \%$. A study by Milentijevic et al ${ }^{11}$ also show a concordance value of $81 \%$. High concordance value was also found by Chabbra et $\mathrm{al}^{8}$ and Bhargava et al.12 Concordance between cytologic and histologic grading was observed more frequently in purely invasive carcinomas (85\%). ${ }^{6}$ This study was close to these studies. However, Dash et $\mathrm{al}^{7}$ and Gayathri et al showed concordance of $77.5 \%$. Sinha et $\mathrm{al}^{13}$ showed a concordance rate of $69.5 \%$.

Low concordance in these studies was probably due to the inclusion of patients receiving chemotherapy and inclusion of cases diagnosed as atypical ductal hyperplasia and in situ carcinomas in cytological samples. In the present study, the patients receiving neoadjuvant chemotherapy were excluded and only cases showing definitive features of ductal carcinoma in both cytology samples and histologic specimens were included. This explains the high concordance between the grading systems.

In this study, association between cytological grading and histological grading system was highest among Grade 2 tumours (91.3\%) followed by Grade 3 (90.9\%) and least with Grade 1 (75\%). Sinha et al ${ }^{13}$ states that Grade 3 tumours showed a greater concordance. Jayaram et al ${ }^{14}$ states that concordance was found to be higher among Grade 3 tumours (83.3\%) and low among Grade 1 tumours (75\%). These studies coincide with the present study. Khan et al ${ }^{10}$ states that higher concordance was found among Grade 1 tumours (92.3\%). Several studies states that concordance was high among high-grade tumours. The present study also supports this view. Out of the seven discordant cases, four of Grade 1 tumours were upgraded. Among two discordant Grade 2 
tumours, one was upgraded to Grade 3 and the other was downgraded to Grade 1 . The one discordant Grade 3 tumour was downgraded. The reason for the upgrading of tumours may be due to sampling errors in large size tumours and heterogeneous tumours. As orientation of the tumour cannot be made more accurate clinically, multiple passes in FNAC were aimed at the central portion of the tumour neglecting the active periphery. In histologic specimens, the infiltrating edge of the tumour was correctly assessed and multiple blocks were made from that site to assess the grade. Another reason of the upgrading of tumours may be due to inadequate samples. The low percentage of concordance in Grade 1 tumours was due to the fact that these low-grade tumours go for a higher grade in the time lag between FNAC procedure and mastectomy, if there is long time gap between the two procedures. One of the reasons for downgrading of tumours may be due to the fact that only nuclear features were taken into consideration while grading the cytologic samples. Tubule formation and mitotic count were not included. There may be subjective variability among the observers, which explains the discrepancies.

Regarding the association between the cytological grade and lymph node status, node positivity was seen in $37.5 \%$, $52.17 \%$ and $72.7 \%$ of Grade 1, 2 and 3 tumours respectively. In a study by Khan et al,10 lymph node metastasis were seen in $15.4 \%$ of Grade $1,55.6 \%$ of Grade 2 and $83.3 \%$ of Grade 3 tumours. Most of the tumours in the present study with more than four positive lymph nodes were Grade 3 tumours. In a study by Robles et al ${ }^{6}$ lymph node metastasis was observed in $8.3 \%$ of Grade $1,64.1 \%$ of Grade 2 and $88 \%$ of Grade 3 tumours. In a study by Dash et al, $74.2 \%$ of Grade 3 tumours showed nodal metastasis in contrast to $27 \%$ in Grade 1 tumours. Our study correlates more with the study by Dash et al. ${ }^{7}$ The correlation between the cytological grade and lymph node status was not statistically significant.

Other demographic factors like age and morphological factors like tumour size and tumour location were also studied. Regarding the age of the patient, most of the tumours were in the post-menopausal age group (52\%). Regarding the tumour size majority of tumours $(60 \%)$ were $\mathrm{T}_{2}$ tumours (2 $5 \mathrm{~cm}$, TNM staging). Since the size of the tumour was an independent time dependent prognostic factor, an attempt was made to correlate tumour size with the cytological grade. No statistical significance was obtained $(p>0.05)$. Similar results were observed in a study by Kim et $\mathrm{al}^{4}$ and Gayathri et al.

Regarding tumour location, most of the tumours were located in outer quadrant accounting for $50 \%$. However, most of the Grade 3 tumours were located in the central quadrant with $54.54 \%$. A study by Harzah et $\mathrm{al}^{15}$ shows that tumours in periareolar location are associated with poor prognosis. In the present study, tumours located in the inner quadrant (60\%) show more lymph node positivity than other quadrant tumours. This is in contrast to all other studies where inner quadrant tumours show lower rate of lymph node positivity. Similar to the present study, Reger et al also showed no correlation between the tumour location and lymph node status.

\section{CONCLUSION}

Due to the increasing incidence of breast cancer in recent years, the role of FNAC in the early identification of breast cancer has been tremendously increased. This present study evaluates prognostic factors in cytological samples. Of the various prognostic factors, cytological grading is the one which is more feasible and reproducible. The histological grade is one of the well-known morphological prognostic factor and had been included in all reports of breast malignancies. This study shows a significant correlation between the cytologic grade and histologic grade. This indicates that cytological grade predicts the tumour aggressiveness.

So, it was concluded that the cytological grading should be included in all FNAC reports, so that appropriate decision regarding the preoperative neoadjuvant chemotherapy can be made and overtreatment of low-grade cancers has been avoided. In this study, cytological grading did not correlate with the lymph node status. When this correlation has been proved, the level of lymph node resection can be planned preoperatively. In spite of adequate efforts made to reduce the distortion in estimates, there is some degree of measurement bias and interobserver variability in this grading system.

This present study suggests the use of ancillary techniques like nuclear morphometry, immunohistochemistry for hormone receptors and proliferative indices done on cytological samples for further evaluation of the tumour biology and aggressiveness.

\section{REFERENCES}

[1] Lester SC. The breast. In: Robbins and Cotran, Pathologic basis of disease. Kumar V, Abbas AK, Fausto N, (eds). $7^{\text {th }}$ edn. Philadelphia: WB Saunders Company Elsevier Inc, 2004:1129-31.

[2] Khan MZ, Haleem A, Al Hassani $\mathrm{H}$, et al. Cytopathological grading, as a predictor of histopathological grade in ductal carcinoma (NOS) of breast on air dried Diff-Quick smears. Diagnostic Cytopathology 2003;29(4):185-93.

[3] Masood S. Prognostic factors in breast cancer: use of cytologic preparations. Diagn Cytopathol 1995;13(5):388-95.

[4] Kim YB, Hwang TS, Kim JM, et al. Correlation of tumor grade and other prognostic factors in invasive breast carcinoma. Journal of Korean Cancer Association 1994;26(6):926-36.

[5] Say CC, Donegan WL. Invasive carcinoma of the breast: prognostic significance of tumor size and involved axillary lymph nodes. Cancer 1974;34(2):468-71.

[6] Robles Frias A, Gonzolez-C $\alpha$ mpora R, Martinez-Parra D, et al. Robinson cytologic grading of invasive ductal breast carcinoma: correlation with histologic grading and regional lymph node metastasis. Acta Cytol 2005;49(2):149-53.

[7] Dash A, Mohanty R. Aspiration smear pattern as a predictor of biologic behavior in breast carcinoma. J Cytol 2005;22(1):19-2.

[8] Chhabra S, Singh PK, Agarwal A, et al. Cytological grading of breast carcinoma-a multivariate regression analysis. J Cytol 2005;22:62-5.

[9] Wani FA, Bhardwaj S, Kumar D, et al. Cytological grading of breast cancers and comparative evaluation of two grading systems. J Cytol 2010;27(2):55-8. 
[10] Khan N, Afroz N, Rana F, et al. Role of cytologic grading in prognostication of invasive breast carcinoma. J Cytol 2009;26(2):65-8.

[11] Milentijevic MJ, Ilić R, Milentijević I. Cytological grading of breast carcinoma with histological correlation. J BUON 2005;10(2):251-6.

[12] Bhargava V, Jain M, Agarwal K, et al. Critical appraisal of cytological nuclear grading in carcinoma of the breast and its correlation with ER/PR expression. J Cytol 2008;25(2):58-61.
[13] Sinha S, Sinha N, Bandyopadhyay R, et al. Robinson's cytological grading on aspirates of breast carcinoma: correlation with bloom Richardson's histological grading. J Cytol 2009;26(4):140-3.

[14] Jayaram G, Elsayed EM. Cytologic evaluation of prognostic markers in breast carcinoma. Acta Cytol 2005;49(6):605-10.

[15] Hazrah P, Dhir M, Gupta SD, et al. Prognostic significance of location of the primary tumor in operable breast cancer. Indian J Cancer 2009;46(2):139-45. 